\title{
REVERSIBLE SKEW GENERALIZED POWER SERIES RINGS
}

\author{
A. R. NASR-ISFAHANI
}

(Received 6 March 2011)

\begin{abstract}
In this note we show that there exist a semiprime ring $R$, a strictly ordered artinian, narrow, unique product monoid $(S, \leq)$ and a monoid homomorphism $\omega: S \longrightarrow \operatorname{End}(R)$ such that the skew generalized power series ring $R[[S, \omega]]$ is semicommutative but $R[[S, \omega]]$ is not reversible. This answers a question posed in Marks et al. ['A unified approach to various generalizations of Armendariz rings', Bull. Aust. Math. Soc. 81 (2010), 361-397].
\end{abstract}

2010 Mathematics subject classification: primary 16S99; secondary 16S36, 16 U80.

Keywords and phrases: skew generalized power series ring, semicommutative, reversible.

\section{Introduction}

Let $(S, \leq)$ be a partially ordered set. Then $(S, \leq)$ is called artinian if every strictly decreasing sequence of elements of $S$ is finite, and $(S, \leq)$ is called narrow if every subset of pairwise order-incomparable elements of $S$ is finite. A monoid $S$ equipped with an order $\leq$ is called an ordered monoid if for any $s_{1}, s_{2}, t \in S, s_{1} \leq s_{2}$ implies $s_{1} t \leq s_{2} t$ and $t s_{1} \leq t s_{2}$. Moreover, if $s_{1}<s_{2}$ implies $s_{1} t<s_{2} t$ and $t s_{1}<t s_{2}$, then $(S, \leq)$ is said to be strictly ordered. Let $R$ be a ring, $(S, \leq)$ a strictly ordered monoid and $\omega: S \longrightarrow \operatorname{End}(R)$ a monoid homomorphism. For $s \in S$, let $\omega_{s}$ denote the image of $\mathrm{s}$ under $\omega$. Let $A$ be the set of all functions $f: S \longrightarrow R$ such that the $\operatorname{support} \operatorname{supp}(f)=$ $\{s \in S: f(s) \neq 0\}$ is artinian and narrow. Then for any $s \in S$ and $f, g \in A$ the set

$$
X_{s}(f, g)=\{(x, y) \in \operatorname{supp}(f) \times \operatorname{supp}(g): s=x y\}
$$

is finite. Thus one can define the product $f g: S \longrightarrow R$ of $f, g \in A$ as follows:

$$
(f g)(s)=\sum_{(x, y) \in X_{s}(f, g)} f(x) \omega_{s}(g(y))
$$

(by convention, a sum over the empty set is 0 ). With pointwise addition and multiplication as defined above, $A$ becomes a ring, called the ring of skew generalized

This research was in part supported by a grant from IPM (No. 89160029). This research was partially supported by the Center of Excellence for Mathematics, University of Isfahan.

(c) 2011 Australian Mathematical Publishing Association Inc. 0004-9727/2011 \$16.00 
power series with coefficients in $R$ and exponents in $S$, denoted by $R[[S, \omega, \leq]]$ (or by $R[[S, \omega]]$ when there is no ambiguity concerning the order) (for more details, see [2]). Special cases of the skew generalized power series construction include skew polynomial rings, skew power series rings, skew Laurent polynomial rings, skew group rings and Mal'cev-Neumann Laurent series rings.

Let $R$ be a ring, $(S, \leq)$ a strictly ordered monoid and $\omega: S \longrightarrow \operatorname{End}(R)$ a monoid homomorphism. A ring $R$ is called $(S, \omega)$-Armendariz if whenever $f g=0$ for $f, g \in$ $R[[S, \omega]]$, then $f(s) \omega_{s}(g(t))=0$ for all $s, t \in S$. Marks et al. in [1] introduced and investigated the notion of $(S, \omega)$-Armendariz rings and studied some property of this class of rings.

A ring $R$ is called reduced if it contains no nonzero nilpotent elements, reversible if for all $a, b \in R, a b=0$ implies $b a=0$, and semicommutative if $a b=0$ implies $a R b=0$ for each $a, b \in R$. It is known that each reduced ring is reversible and each reversible ring is semicommutative, but the converse not true in general. Marks et al. in [1] characterized when a skew generalized power series ring is reduced or semicommutative, and obtained a partial characterization for it to be reversible. They proved that for a strictly ordered monoid $(S, \leq)$, a monoid homomorphism $\omega: S \longrightarrow$ $\operatorname{End}(R)$ and an $(S, \omega)$-Armendariz $S$-compatible $\operatorname{ring} R, R[[S, \omega]]$ is semicommutative if and only if $R$ is semicommutative. They also showed that for a strictly ordered monoid $(S, \leq)$ which is an artinian, narrow, unique product (a.n.u.p., see [1, Definition 4.11]) and a monoid homomorphism $\omega: S \longrightarrow \operatorname{End}(R), R[[S, \omega]]$ is reduced if and only if $R$ is semiprime and the ring $R[[S, \omega]]$ is reversible. Marks et al. in [1] posed the following question (Question 4.14): 'Suppose $R$ is a semiprime $\operatorname{ring},(S, \leq)$ is a strictly ordered a.n.u.p. monoid and $\omega: S \longrightarrow \operatorname{End}(R)$ is a monoid homomorphism. If the skew generalized power series ring $R[[S, \omega]]$ is semicommutative, must $R[[S, \omega]]$ be reversible (and therefore reduced)?'.

In this note we provide a semiprime ring $R$, a strictly ordered a.n.u.p. monoid $(S, \leq)$ and a monoid homomorphism $\omega: S \longrightarrow \operatorname{End}(R)$ such that the skew generalized power series ring $R[[S, \omega]]$ is semicommutative but $R[[S, \omega]]$ is not reversible. This gives a negative answer to the question posed by Marks et al. We also prove that for a semiprime ring $R$, a strictly ordered a.n.u.p. monoid $(S, \leq)$ and a monoid homomorphism $\omega: S \longrightarrow \operatorname{End}(R), R[[S, \omega]]$ is reversible if and only if $R[[S, \omega]]$ is semicommutative and $\omega_{s}$ is injective for each $s \in S$.

\section{Main results}

Let $R$ be a ring and $\alpha$ be a ring endomorphism. We denote by $R[x ; \alpha]$ the skew polynomial ring whose elements are the polynomials over $R$, addition is defined as usual, and multiplication is subject to the relation $x a=\alpha(a) x$ for any $a \in R$.

ExAmple 2.1. Let $K$ be a field, $R=K[x], S=\mathbb{N} \cup\{0\}$ with the usual addition and trivial order. $\alpha: R \rightarrow R$ given by $\alpha(f(x))=f(0)$ is a ring homomorphism and so $\omega: S \longrightarrow$ $\operatorname{End}(R)$ given by $\omega(1)=\alpha$ is a monoid homomorphism. We have $R[[S, \omega]] \cong R[y ; \alpha]$. 
We show that $R[y ; \alpha]$ is semicommutative but not reversible. Assume that $f=f_{0}+$ $f_{1} y+\cdots+f_{n} y^{n}, g=g_{0}+g_{1} y+\cdots+g_{m} y^{m} \in R[y ; \alpha]$ is such that $f g=0$. By induction on $\operatorname{deg}(g)=m$ we show that $f R[y ; \alpha] g=0$. If $m=0$ then $f_{n} \alpha^{n}\left(g_{0}\right)=0$. Since $R$ is a domain, we have $\alpha^{n}\left(g_{0}\right)=0$ and so $g_{0} \in(x)$, where $(x)$ is the ideal generated by $x$ in $R$. We also have $f_{0} g_{0}=0$. If $g_{0}=0$ then $f R[y ; \alpha] g=0$. If $g_{0} \neq 0$ then $f_{0}=0$ and so $f R[y ; \alpha] g=0$.

Now assume inductively that the assertion is true for all $g \in R[y ; \alpha]$ with $\operatorname{deg}(g)<m$. Since $f g=0$, we have $f_{n} \alpha^{n}\left(g_{m}\right)=0$ and so $g_{m} \in(x)$. Also we have $f_{0} g_{0}=0$. If $f_{0} \neq 0$ then $g_{0}=0$ and so $f_{0} g_{1}=0$. Thus $g_{1}=0$ and, by the same argument, in this case we have, for each $i, g_{i}=0$. Then $f R[y ; \alpha] g=0$ and the result follows.

Now assume that $f_{0}=0$. Since $g_{m} \in(x)$ and $f_{0}=0$ then $f R[y ; \alpha] g_{m} y^{m}=0$ and so $f\left(g_{0}+g_{1} y+\cdots+g_{m-1} y^{m-1}\right)=0$. By the induction hypothesis,

$$
f R[y ; \alpha]\left(g_{0}+g_{1} y+\cdots+g_{m-1} y^{m-1}\right)=0 .
$$

Thus we have $f R[y ; \alpha] g=0$ and the result follows. In $R[y ; \alpha]$ we have $y x=\alpha(x) y=0$ but $x y \neq 0$. Thus $R[y ; \alpha]$ is not reversible.

Let $R$ be a semiprime ring. In the next theorem we provide a necessary and sufficient condition for a skew generalized power series ring $R[[S, \omega]]$ to be reversible.

THeOREM 2.2. Let $R$ be a semiprime ring, $(S, \leq)$ a strictly ordered a.n.u.p. monoid and $\omega: S \longrightarrow \operatorname{End}(R)$ a monoid homomorphism. Then $R[[S, \omega]]$ is reversible if and only if $R[[S, \omega]]$ is semicommutative and $\omega_{s}$ is injective for each $s \in S$.

Proof. If $R[[S, \omega]]$ is reversible, then by [1, Theorem 4.12] $\omega_{s}$ is injective for each $s \in$ $S$. Now assume that $R[[S, \omega]]$ is semicommutative and $\omega_{s}$ is injective for each $s \in S$. Let $s \in S, \omega_{s} \in \operatorname{End}(R)$ and $a \in R$ such that $a \omega_{s}(a)=0$. Since $R$ is semiprime and semicommutative, then $R$ is a reduced ring and so $\omega_{s}(a) a=0$. Thus by [1, Lemma 4.4], we have $\omega_{s}(a) \omega_{s}(a)=0$. Then $a^{2}=0$ and so $a=0$. Thus for each $s \in S, \omega_{s}$ is a rigid endomorphism. Then, by [1, Theorem 4.12], $R[[S, \omega]]$ is reversible and the result follows.

\section{References}

[1] G. Marks, R. Mazurek and M. Ziembowski, 'A unified approach to various generalizations of Armendariz rings', Bull. Aust. Math. Soc. 81 (2010), 361-397.

[2] R. Mazurek and M. Ziembowski, 'On von Neumann regular rings of skew generalized power series', Comm. Algebra 36(5) (2008), 1855-1868.

A. R. NASR-ISFAHANI, Department of Mathematics, University of Isfahan, PO Box 81746-73441, Isfahan, Iran and

School of Mathematics, Institute for Research in Fundamental Sciences (IPM), PO Box 19395-5746, Tehran, Iran e-mail: a_nasr_isfahani@yahoo.com 